\title{
Desarrollo de la metodología en ciencias sociales en América Latina: posiciones teóricas y proyectos de sociedad
}

\section{Fernando Cortés*}

\author{
Perfiles Latinoamericanos | Ensayo
}

vol. 23 | núm. 45 | 2015

pp. $181-202$

\begin{abstract}
Resumen
La metodología de las ciencias sociales suele conceptuarse como un campo disciplinario provisto de su propia lógica interna. En este ensayo se muestra que a pesar de ello, al examinar las disputas metodológicas que han tenido lugar en América Latina en los últimos sesenta ańos (así como las teóricas), se observa que han sido influidas por las luchas políticas por imponer proyectos de sociedad alternativos. Política, teoría y metodología han estado fuertemente imbricadas. Se plantea que hasta fines de los sesenta la metodología era equivalente a técnicas de encuesta. En los setenta y parte de los ochenta se redefinió por la preocupación de comprender el cambio estructural desde una epistemología con fuerte acento marxista. A raíz de las crisis de los ochenta y las consecuentes restricciones presupuestarias sobre la investigación académica se ha observado una tendencia a limitar la metodología a un conjunto de herramientas y técnicas de investigación, y a discutir conceptos desprendidos de sus cuerpos teóricos, ocultando así la diversidad de enfoques y propuestas políticas.
\end{abstract}

\begin{abstract}
The methodology of the social sciences is often conceptualized as a disciplinary field equipped with its own internal logic. This article shows that in spite of this, when examining methodological disputes (and theoretical as well) that have taken place in Latin America in the last sixty years shows that have been influenced by political struggles to impose alternative social projects. Politics, theory and methodology have been closely intertwined. It is argued that until the late sixties methodology was equivalent to survey techniques. In the seventies and part of the eighties there was a concern to understand the structural change with a strong Marxist epistemology accent. Following the crisis of the eighties and the consequent budgetary restrictions on academic research there has been a tendency to limit the methodology to a set of research tools and techniques, and discuss concepts detached form their theoretical bodies, hiding the diversity of approaches and policy proposals.
\end{abstract}

Palabras clave: metodología de la investigación social, historia de las ideas, técnicas de investigación social y relación teoría-metodología.

Keywords: methodology of social research, history of ideas, social research techniques and relationship theory-methodology.

* Profesor emérito de la Flacso México. Investigador del PUED, unam. 


\section{Introducción}

- 1 ctualmente, el término metodología es polisémico. Cuando en algún país de América Latina se ofrece un curso de metodología, su contenido puede abarcar desde técnicas estadísticas para analizar datos, hasta la exposición y discusión de temas epistemológicos abstrusos, pasando por cursos de construcción de cuestionarios, métodos para realizar observación directa o participante, modelos estadísticos avanzados, lógica de la investigación, discusiones acerca de la naturaleza supuestamente irreductible de los denominados métodos cualitativos y cuantitativos, entre otros. Por una parte, en la docencia se observa bastante autonomía entre temas teóricos, técnicos y metodológicos; y, por otra, en la investigación no suelen hacerse explícitos los vínculos que guardan las posiciones teóricas, la metodología y las técnicas de investigación, con los problemas que viven nuestras sociedades.

En este escrito me propongo, en primer lugar, construir una explicación del porqué de la polisemia del término metodología en las ciencias sociales latinoamericanas. Una explicación que descansa, en gran parte, en la relación que, en el pasado, han tenido los procesos de transformación social y de lucha política con los problemas teóricos y metodológicos que han preocupado a las ciencias sociales en estas latitudes y longitudes. En segundo lugar, intentaré mostrar que esta manera particular de pensar sobre el tipo y dinámica de los problemas metodológicos nos ayuda a centrar nuestros intereses con respecto a la lucha ideológica que se despliega en torno a los procesos sociales que vivimos actualmente.

\section{La década de los sesenta}

No es de mi interés hacer un recuento exhaustivo acerca de la evolución de la metodología de las ciencias sociales en América Latina, remontándome a la época en que estas disciplinas no se habían profesionalizado aún, sino limitarme a tratar algunos temas —a mi juicio, centrales - que muestran las relaciones entre los problemas que ha abordado la metodología, los cambios en los paradigmas teóricos y el acontecer social y político en la época moderna, época que arbitrariamente dato a partir de la década de 1960.

Hasta el primer quinquenio de los sesenta, predominaron — sin contrapeso en el plano conceptual_ el funcionalismo estructural parsoniano y las teorías de alcance medio de Robert Merton, así como las técnicas de encuesta (survey) en el plano metodológico. Teoría y metodología se combinaban armónicamente con los instrumentos que en esa época proporcionaba la estadística. 
La información empírica que permitía contrastar las hipótesis de las investigaciones sociológicas se obtenía preferentemente a través de muestras que usaban cuestionarios como instrumentos de recopilación de información, por lo que para los científicos sociales de esos años, era crucial comprender la teoría del muestreo así como sus diversas formas de aplicarla. Sin embargo, para llegar hasta ese punto, era, y es, necesario disponer de un buen nivel de conocimiento de la estadística descriptiva y de la teoría de probabilidades.

Una síntesis de lo que se entendía por metodología en esos años se encuentra en Teoría y métodos de la investigación social, de Johan Galtung, profesor de metodología de la Flacso en Santiago de Chile, publicado por la Editorial Universitaria de Buenos Aires en 1966, y que apareció en inglés al año siguiente con el título Theory and Methods of Social Research, editado por la Columbia University en Estados Unidos.

En los años sesenta, gran parte de los desarrollos metodológicos de punta en la investigación social se concentraban en el tratamiento de la causalidad en la investigación no experimental, etapa iniciada por el señero trabajo de Hubert M. Blalock, Causal Inference in Nonexperimental Research, publicado en 1964 por Chapell Hill, en North Carolina, obra que culminaba el tratamiento estadístico inaugurado por el genetista poblacional Wright en 1934, y que incluía el artículo de Lazarsfeld sobre el análisis de covarianzas, que se presentó en público por primera vez en 1946 en un congreso de la Sociedad Americana de Sociología en Cleveland, y un trabajo de Simon publicado en 1957. En Francia, fue notable la contribución de Raymond Boudon (profesor de Metodología de la investigación y Sociología de la Educación en la maestría en Sociología de la Flacso), quien publicó en la editorial Plon de París, en 1968, L'Analyse Mathematique des Faits Socieaux, cuyo tercer capítulo está dedicado al análisis de la causalidad con datos generados por observación.

En el segundo quinquenio de los sesenta, el quehacer de los científicos sociales vivió movimientos de mar profundo con la irrupción del marxismo en las aulas de nuestras universidades. En esa época se desató una lucha paradigmática en la que se entreveraron las discusiones propiamente académicas, los procesos de transformación social y política, las disputas ideológicas y el sentido que debía darse a esas transformaciones para construir sociedades más justas. El camino que abrió la revolución cubana enseñó a los jóvenes de América Latina que sí era factible emprender cambios de fondo en nuestras sociedades.

Dejando a un lado las turbulencias sociales, políticas, ideológicas y culturales de la época, y mirado en retrospectiva con el reposo que da el paso del tiempo, se puede decir que eran dos los grandes temas que atravesaban el quehacer de las ciencias sociales de entonces: 1) la lucha entre el paradigma dominante de la modernización y el emergente de la dependencia, que se reflejaba en la disputa 
política que sostenían esencialmente la izquierda y la centro izquierda, en la que predominaban los partidos con orientación cristiana, y 2) las discusiones teóricas dentro de la izquierda referidas a la conquista del poder, que surgieron después de la victoria de la revolución cubana, cuyo amplio espectro se extendía desde la posición que proponía la vía armada, hasta la que planteaba el camino de las urnas.

Mientras dichos temas eran el centro de la disputa en las aulas, los profesores dictaban clases sobre la teoría de los sistemas sociales de Talcott Parsons, las teorías de alcance medio de Robert Merton, o la economía del bienestar. Pero en los salones de clases, debajo de sus pupitres, los estudiantes leían un mimeografiado (aún no existían las fotocopias) — que sólo era legible después de un arduo trabajo de rellenado de las letras faltantes debido al desgaste de la matriz-, obra que posteriormente sería publicada por siglo XxI con el título Dependencia y desarrollo en América Latina de Fernando Henrique Cardoso y Enzo Faletto; este último, chileno del barrio Nuñoa, donde nació y murió.

El enfoque ${ }^{1}$ de la dependencia — nacido en el corazón mismo de CEPAL-, asiento institucional de la teoría del desarrollo económico que predominó en la época, y que se elaboró bajo la conducción de Raúl Presbich — conocida en el ambiente académico como la teoría cepalina- hizo ver, por una parte, que la comprensión del problema del desarrollo no sólo debía analizarse desde el ángulo económico, sino que también había de considerar su aspecto social; mientras que, por otra, que se requerían de nuevas metodologías para enfrentar el reto explicativo.

Surge así la discusión en torno a la investigación multidisciplinaria —circunscrita inicialmente al campo de la economía, la sociología y la ciencia política-, en medio de profundos procesos de transformación social y política. Es esta la época en la que se hizo claro que no debía perderse de vista que los problemas sociales no reconocen las fronteras disciplinarias arbitrarias impuestas por el hombre en su afán de conocer. La realidad aún no se ha enterado que para estudiarla la hemos dividido en disciplinas.

En un intento por adecuar el conocimiento metodológico y estadístico acumulado a las nuevas circunstancias que vivían las ciencias sociales de la época, Manuel Castells y Fernando Cortés se asociaron para analizar los vínculos entre la teoría, las hipótesis y las técnicas estadísticas. Como resultado de este

1 Es habitual que se hable de la teoría de la dependencia, cuando en realidad hubo varias. En este texto, dedicado a tratar principalmente temas metodológicos, destaco el papel de la versión Cardoso-Faletto. Además, no uso la palabra "teoría" sino "enfoque" por respeto a las ideas de Enzo, para quien "las teorías" deberían no sólo ser un conjunto coherente y articulado de enunciados abstractos, sino incluir también los métodos y las técnicas que permiten hacer observables los conceptos en el plano de la experiencia. 
trabajo conjunto, escribieron el artículo "Análisis causal y técnicas estadísticas en la investigación sociológica", publicado por la Facultad Latinoamericana de Ciencias Sociales en 1968.

Nanterre, en 1968, diseminó por América Latina el marxismo estructuralista de origen francés. Para leer el capital, de Louis Althusser, fue traducido al español; sus discípulos, que llegaron a América Latina evitando la persecución política, trajeron una propuesta teórica y metodológica que cayó en terreno fértil.

Se propagó el dictum que afirmaba que "la estructura determina, en última instancia, la superestructura", de modo que bastaba con cambiar las relaciones sociales de producción para desencadenar procesos irreversibles de transformación social. ${ }^{2}$

Dos de las contribuciones más importantes de los franceses a la discusión teórico-metodológica — como se decía en la época— - fue poner el acento sobre los modos de producción y su articulación en formaciones sociales concretas, así como en la idea de una cierta sucesión histórica de los modos de producción dominantes, nunca explícitamente reconocida. Esta perspectiva conceptual, unida a la teoría de la dependencia, originó hacia fines de la década de los sesenta el análisis histórico estructural que tuvo fuerte influencia sobre la mayor parte de las comisiones que trabajaban en el seno del naciente Consejo Latinoamericano de Ciencias Sociales (CLACSO) y en la formación del proyecto PROELCE, impulsado conjuntamente por la Escuela Latinoamericana de Sociología (ELAS) de la Flacso y el Centro Latinoamericano de Demografía (CELADE), que puso en el centro de la discusión el papel de la población en los procesos sociales.

Efectivamente, alrededor de los años setenta, el análisis histórico-estructural se extiende desde la sociología y la ciencia política a la demografía. Su origen proviene de un convenio firmado entre el CELADE y la ELAS, del cual surge el Programa ELAS-CELADE (PROELCE) que, encabezado por Susana Torrado, centró su interés en las determinantes sociales de los fenómenos demográficos. La idea dominante en la época era que a cada modo de producción le correspondía su propia ley de población. La migración dejó de analizarse como una decisión individual y empezaron a estudiarse vis à vis los requerimientos diferenciales de mano de obra según los sistemas productivos; mientras que las transformaciones en los patrones

2 Sin embargo, la historia de Chile alrededor de 1973 enseñó que el traspaso de la propiedad de los medios de producción a los trabajadores y la nacionalización de las riquezas naturales en manos de capital extranjero, no se tradujeron en un cambio revolucionario irreversible, sino más bien en una violenta vuelta atrás en el tiempo. Este hecho obligó a la reflexión sobre lo acontecido: algunos reaccionaron invirtiendo el acento al postular el predominio de la superestructura sobre la estructura o la autonomía relativa de la superestructura — posición que encontró aval teórico en Antonio Gramsci-, otros se plantearon la negación del peso de la teoría y la búsqueda descarnada de la realidad, posición que se aproxima en lo esencial al empirismo lógico de la década de 1920. 
migratorios fueron conceptuadas como respuesta a los cambios en las relaciones sociales de producción. Del mismo modo, los estudios buscaron establecer vínculos entre los modos de producción y los patrones de fecundidad y mortalidad.

Otro de los temas esenciales para el desarrollo del conocimiento metodológico que llegó de Francia fue la idea de que el dato se construye. Hoy se puede afirmar, sin temor a equivocarse, que, en los sesenta, la sociología y la ciencia política académicas tendían a no discutir sus fundamentos filosóficos. El positivismo lógico ejercía un dominio legítimo; se hallaba ampliamente extendida la idea de que investigar consistía en identificar regularidades en los datos, que a su vez eran la piedra angular del conocimiento válido en la medida en que eran o representaban la realidad. Plantear en este medio que el dato se construye, y que se construye a partir de conceptos teóricos, debería haber provocado un sismo, pues se hacía explotar una bomba en los cimientos de la fábrica de conocimientos.

Sin embargo, los cambios sociales, las nuevas correlaciones políticas que afectaban a varios países del Cono Sur, y el desmoronamiento de la teoría, la metodología y las técnicas de investigación —incluida la estadística-, ante los embates del enfoque de la dependencia, el surgimiento del método histórico estructural y el predominio del marxismo académico, opacaron las consecuencias del dictum "el dato se construye".

No obstante, bajo el imperio de este dictum, acicateado por la propuesta de las Naciones Unidas para crear un sistema de estadísticas sociales y demográficas equivalente al sistema de cuentas nacionales, se inició en el PROELCE una línea de investigación en torno a la idea de que el dato no está dado, sino que se construye. La respuesta a la propuesta de las Naciones Unidas involucró aspectos epistemológicos, teóricos e ideológicos. En las ciencias sociales de América Latina, implícita o explícitamente, se abrazaba la idea empirista de que los datos están dados. La nueva idea que había llegado del viejo continente caló profundo en la comunidad académica de la época; en la medida en que la discusión fue avanzando en extensión y profundidad, se fue haciendo cada vez más claro que la óptica teórica es un ingrediente importante en la construcción del dato. Con base en esta nueva conceptuación, se sostuvo que la propuesta de las Naciones Unidas tenía al estructural funcionalismo como marco conceptual y que, por lo tanto, podía verse como una forma de perpetuar — en lugar de cambiarlas estructuras sociales. Además, a través de este argumento, las discusiones sobre la naturaleza del dato pasaron a formar parte del debate político ilustrado.

La creación del grupo de trabajo del CLACso "Sistema de Estadísticas Sociales y Demográficas”, originó una amplia producción para ilustrar, a través de investigaciones empíricas, las diversas formas como se construye el dato. En esta vasta producción, se debe destacar el trabajo de Susana Torrado, Emilio de Ípola, José 
María Carrón y Arturo León, quienes reanalizaron el censo chileno de 1970 a partir de las categorías de la interpretación althousseriana de El Capital. El resultado de esta investigación mostró que, a partir de la misma fuente de datos, surgían dos imágenes de país: una que era producto de aplicar las categorías tradicionales, basadas en los atributos de las personas cuyo tratamiento dividía a la población en estratos sociales, y otra muy distinta, que emergía de centrar la atención en las clases sociales inscritas en las relaciones sociales de producción. ${ }^{3}$

Hacia finales de la década de los sesenta, la preocupación se centró en cómo pensar y entender el cambio estructural. Este nuevo foco de la preocupación enraíza en la fuerte disputa política que irrumpe después del triunfo de la revolución cubana, disputa que abre la posibilidad histórica en varios países de América Latina - y en particular en Chile- de que los partidos políticos con orientación marxista lleguen al poder. Esto trajo como consecuencias: 1) que la corriente sociológica estructural-funcionalista abocada a estudiar la estabilidad de los sistemas sociales fuese desafiada y expulsada de las aulas en las principales escuelas de sociología y ciencia política. Algo similar ocurre - aunque en menor medida- en las escuelas de economía, donde se sustituye la denominada "teoría económica" por la "economía política" inspirada en El Capital. Y 2) el cuestionamiento de las técnicas de análisis de datos orientadas a tratar información recabada mediante encuestas por muestreo aplicadas a las personas, que originaba análisis de secciones cruzadas que no guardaban relación con el interés en estudiar procesos diacrónicos.

Debe quedar claro que hacia el último quinquenio de los sesenta, la enseñanza del estructural funcionalismo y de las técnicas de encuesta (survey) no respondía a las inquietudes sociales de la juventud de la época: primaba en ellos el interés por conocer los condicionantes del cambio estructural. Este interés no era sólo académico: se inscribía en la política contingente. Por esos años, era valorizada la participación política a través de los partidos y los académicos no escapábamos al predominio de este valor. Detrás de la preocupación por el cambio estructural, estaba la idea de generar conocimientos para transformar la sociedad, cada quién estaba motivado por el interés de provocar los cambios que propugnaba su praxis política.

Adam Przeworski, además de enseñar teoría política y análisis de la causalidad en la maestría en Sociología de la Flacso, escribía en Santiago de Chile

3 La historia tiene misterios. La estadística, ayer como hoy, tiene una fuerte influencia de la obra de Karl Popper, autor que ha sido calificado como neopositivista. Esta corriente filosófica, ya en la década de los treinta, planteaba la incapacidad del sujeto para aprehender directamente el objeto; la relación sujetoobjeto era mediada, el objeto era construido. Sin embargo, esta idea llegó a través de los desplazados de Nanterre. Tal vez ello se deba a la casi nula discusión epistemológica y la muy pronunciada preocupación por las técnicas de investigación y la estadística que predominaban por aquella época. 
la versión final del libro The Logic of Comparative Social Inquiry, el cual saldría publicado en 1972 por John Wiley. Al mismo tiempo, y junto con Fernando Cortés, Przeworski iniciaba las investigaciones sobre el análisis de sistemas sociales, que culminarían en la publicación de System Analysis for Social Scientists, obra a la que se agregó en calidad de coautor a John Sprague, profesor de la Washington University en Saint Louis Missouri. El texto sería publicado en 1974 por John Wiley. Uno de sus primeros capítulos, escrito en forma de artículo por Cortés y Przeworski, apareció en el segundo volumen del año 1971 de la Revista Latinoamericana de Ciencias Politicas y Administrativas con el título "Sistemas partidistas, movilización electoral y la estabilidad en sociedades capitalistas", cuyo tema central era la estabilidad —o mirado desde otro punto de vista - el estudio de las condiciones bajo las cuales el sistema político se desestabiliza, generando condiciones favorables para el cambio estructural.

A partir de las nuevas preocupaciones teóricas, emergió la necesidad de enfrentar los nuevos problemas metodológicos que se derivaban del enfoque de la dependencia, que ponía el acento en el análisis histórico. Como ya hemos dicho, la metodología de las ciencias sociales estaba fuertemente dominada por las técnicas de survey, que entre otras cosas, se caracterizaban por ser esencialmente estáticas. Si bien existía ya en la bibliografía la idea de la encuesta de panel, aún estábamos lejos de los desarrollos teóricos, de los métodos de análisis y de las posibilidades de procesamiento de las que disponemos hoy. El estudio estadístico de series de tiempo se reducía a la identificación, diferenciación y cuantificación de la tendencia, las fluctuaciones estacionales y las variaciones cíclicas e irregulares. Los poderosos métodos con que contamos hoy, encapsulados en los programas que procesan las computadoras personales para tratar eventos cronológicos, aún estaban en el futuro. A su salida de la CEPAL, Faletto ingresó a la Flacso con la idea de transformar el enfoque en una teoría. El proyecto fue truncado el 11 de septiembre de 1973 por el golpe de estado en contra del gobierno de Salvador Allende.

\section{El inicio de la metamorfosis. De las dictaduras a la crisis de la deuda externa}

Entre el comienzo de la década de los setenta hasta alrededor de la mitad de la década de los ochenta, ${ }^{4}$ la estadística fue expulsada de los salones de clase y de la investigación social.

4 El límite superior del periodo no es tan nítido como en el anterior, cualquiera que se elija puede ser objeto de controversia, sin embargo, como es bien sabido, toda periodización tiene cierta dosis de arbitrariedad; los procesos sociales suelen no tener límites tan marcados. 
En los primeros años de la década de 1970, los partidos de izquierda de ideología marxista, en América del Sur, tuvieron avances importantes en la conquista del poder político. En el plano de las ciencias sociales, el surgimiento y predominio de la corriente histórico-estructural forzó cambios importantes en los programas de formación de economistas, sociólogos y politólogos. Los teóricos "funcionalistas" pasaron al cajón de los recuerdos de la mano de la economía del bienestar; se impuso en cambio el estudio del marxismo y de Marx. Es la época en que Karl Marx se viste de toga y birrete e ingresa a la Universidad. Las nuevas generaciones de científicos sociales estudiaban desde diferentes ángulos cada una de sus obras; se volvieron clásicos los estudios de los marxistas rusos, entre los que destacaban los trabajos de Lenin, y se leían y discutían con avidez las obras marxistas de tercera generación elaboradas en el seno de la escuela francesa.

La investigación, en consonancia con las disputas políticas por el poder, se concentró en el estudio del cambio estructural, en la sucesión de los modos de producción o en la evolución de las formaciones sociales concretas. El problema central de las ciencias sociales era dar inteligibilidad a los procesos sociales y políticos que estaban aconteciendo y proponer mecanismos para orientarlos hacia objetivos predefinidos. Las preguntas de investigación indagaban sobre unidades de análisis agregadas y revestían un carácter eminentemente histórico. Quedaba en el pasado el interés por el estudio del comportamiento, actitudes, valores, percepciones, etc. de los individuos localizados en un punto del tiempo. El foco de la atención se había desplazado a los procesos de constitución y cambio de los movimientos populares, de la clase obrera o de los campesinos. La naciente sociodemografía estudiaba los flujos migratorios en lugar de la decisión de migrar, la relación entre la fecundidad y las clases sociales, los vínculos entre la dinámica demográfica y las formaciones sociales concretas. Los aires de la época dictaban que el interés de la investigación social debía constreñirse al análisis de la dinámica de la estructura y sólo secundariamente al individuo que habitaba un espacio nacional o regional en una época determinada.

El avance del marxismo, del análisis histórico estructural y el enlace entre la investigación y los procesos de transformación social que estaban acaeciendo, tuvieron incidencia evidente sobre los programas de estudio de la metodología y la estadística social.

Los instrumentos que ponían la metodología y la estadística a disposición de la investigación social de esos años quedaron fuera de foco ante el cambio en el paradigma. No estaban diseñados para ayudar a entender el cambio estructural.

Por otro lado, los nuevos problemas de investigación también provocaron un cambio de contenido en la metodología de las ciencias sociales. Una parte de la exploración en busca de métodos que ayudaran a responder las pregun- 
tas que se formulaba la nueva investigación social, se volcó hacia el estudio de la filosofía de la ciencia y de la epistemología. Otro camino que se ensayó fue la lectura metodológica de las investigaciones realizadas por los autores clásicos.

El muestreo y la inferencia se eliminaron de los programas de la enseñanza de la estadística, así como de cualquier técnica de investigación. La estadística descriptiva se enseñó como parte de cursos de "Fundamentos técnicos de la investigación social" en los cuales se estudiaba la forma en la que se operacionalizaban las relaciones entre los conceptos teóricos en investigaciones calificadas como clásicas en esa época y las de nuevo cuño. ${ }^{5}$

Los golpes de estado acaecidos en América del Sur durante los primeros años de la década de los setenta y la persecución política desatada en contra de los académicos clausuró a piedra y lodo las posibilidades de la docencia y la investigación de las ciencias sociales en varios países, aunque se mantuvo un cierto nivel de investigación académica de bajo perfil a pesar de los riesgos en los que incurrieron los investigadores.

En los países que escaparon a la barbarie militar, se continuaron desarrollando las mismas orientaciones metodológicas pre golpes de estado; al trabajo desarrollado por los profesores investigadores de cada país, se agregó el aporte de los académicos desterrados. Desde el exilio, la preocupación intelectual se volcó sobre la experiencia vivida y el énfasis cambió de lo estructural a lo superestructural. La década de los ochenta estuvo signada por la reflexión acerca del proceso de transformación del Estado y su autonomía relativa. Sin embargo, fueron contados con los dedos de las manos los estudios concretos, más allá de los ensayos especulativos, realizados en la región.

Desde los inicios de los ańos ochenta, fue retomado el problema del cambio estructural en los seminarios sobre epistemología genética encabezados por Rolando García y Juan Carlos Marín, pero esta vez desde la perspectiva de los sistemas complejos en los que la noción de continuidad funcional y discontinuidad estructural (etapas del desarrollo cognitivo) permiten analizar el comportamiento diacrónico de los sistemas, cuyo motor son las contradicciones entre lo que se espera de lo "real" y su "comportamiento" (García, 2000). Esta idea fue desarrollada en el Centro de Epistemología Genética, en Ginebra, y presentada en el libro de Jean Piaget La equilibración de las estructuras cognitivas. Problema central del desarrollo, publicado en español por Siglo XXI en 1978. Esta manera de conceptuar la evolución de los sistemas complejos fue utilizada por Illya Prigogine e Isabel Stengers en La nueva alianza. Metamorfosis de la ciencia, publicado por Alianza Editorial en 1979, y por Jean Piaget y Rolando

5 Nótese que no uso el término de hipótesis teórica pues, en esos años, la palabra hipótesis era "reaccionaria”, tenía un fuerte tufillo funcionalista. 
García en Psicogénesis e historia de la ciencia, publicado originalmente en espanol por Siglo XXI en 1982 y traducido a varios idiomas.

Tomando pie en la idea de que los procesos cognitivos (Piaget), químicos y físicos (Prigogine) y la historia de la ciencia (Kuhn y Piaget y García) son estructuralmente discontinuos pero funcionalmente continuos, se dirigió la atención al proceso de investigación. En la epistemología genética, la relación de conocimiento entre el sujeto y el objeto dista de agotarse en una simple "flecha”, aunque sea bidireccional; lo importante es entender que dicha relación es la misma para todos los seres humanos, sin importar si es un científico, un adulto común, o un niño.

En la introducción al libro Métodos estadísticos aplicados a la investigación en ciencias sociales, publicado por El Colegio de México en 1987, de Fernando Cortés y Rosa María Rubalcava, que a la sazón se desempeñaban como profesores investigadores en la Flacso México y en El Colegio de México, respectivamente, se encuentran los primeros planteamientos sobre el proceso de investigación social basado en los conceptos precedentes. Se abandona la idea de que la investigación discurre rectilíneamente entre la formulación del problema, las hipótesis, su contrastación y la producción de nuevo conocimiento. Se argumenta que la mejor representación gráfica del proceso de investigación sería una espiral en tres dimensiones. En el libro se plantea, y además se ilustra, que el análisis de asociación se puede ver como una técnica estadística que permite confrontar la distribución de los datos que se observarían si fuesen consistentes con la teoría, en relación con los efectivamente observados.

La idea de representar el proceso de investigación con una espiral se fue enriqueciendo de manera tal, que 25 ańos después ha culminado en El helicoide de la investigación. Metodología en tesis de ciencias sociales, publicado por la Flacso México en 2013. Esta es una obra colectiva en que los doctores recién titulados — que han pasado por los cursos de Metodología de la Investigación en Ciencias Sociales impartidos en las maestrías y doctorados de la Flacso, Sede México- reflexionan sobre el proceso de investigación que culminó en sus tesis, apoyados no sólo por un conjunto de profesores sino también por las críticas, señalamientos e intercambio de experiencias con sus propios compañeros de aventura intelectual.

\section{Los últimos treinta años a partir de la crisis de la deuda externa}

A lo largo de la década de los ochenta, tendían a desaparecer los gobiernos de facto en América Latina y a ser sustituidos por otros electos por el voto ciudadano; la democracia electoral se extendía como un tsunami por la región. 
Este avance democrático se combinó con el estancamiento económico. La explosión de la crisis del petróleo en 1982 socavó las bases del crecimiento en América Latina. La "década perdida" se caracterizó, entre otras cosas, por los severos recortes que sufrió el gasto de los gobiernos, al buscar un equilibrio con los ingresos. Ganaba espacio el concepto de "déficit cero" en el balance contable de las cuentas del gobierno; idea que a la vuelta del tiempo sería uno de los componentes de la primera oleada del Consenso de Washington (Williamson, 1990).

Hacia finales de la década de los ochenta y durante los años noventa, la mayoría de los países de la región iniciaron procesos de cambios estructurales tendientes a liberar las fuerzas del mercado; la consigna era "más mercado y menos Estado". A pesar de los pronósticos basados en la "teoría económica" que apostaban a los efectos beneficiosos sobre las economías de América Latina, éstas exhibieron durante los años noventa inestabilidad y ausencia de crecimiento. ${ }^{6}$ Era la "segunda década perdida" consecutiva (Williamson, 2003: 1-6).

La difícil situación económica generalizada se dejó sentir sobre las instituciones de educación superior, afectando la investigación y la docencia. El financiamiento de las investigaciones, proveniente de fundaciones u organismos internacionales ganó en importancia en algunos países y también tendió a centralizarse en consejos nacionales de ciencia y tecnología. Hubo países que crearon becas para complementar salarios en las propias instituciones de educación superior, o bien financiamiento proveniente de organismos externos a ellas creados ex profeso para impulsar y orientar la investigación.

Los escasos recursos se inclinaron y se inclinan actualmente a ser canalizados al estudio de problemas sociales específicos, entre los cuales se privilegia la investigación social empírica. La caída del poder adquisitivo de los salarios de los académicos y los cambios institucionales crearon un contexto cada vez más desfavorable para los estudios especulativos (entendidos como aquellos que se refieren a los procesos y problemas sociales sólo a modo de ejemplo), así como para los genuinamente teóricos que habían gozado de alta retribución y estima social a comienzos de la década de los ochenta.

En este ambiente, la metodología de las ciencias sociales volvió sobre sus pasos y recuperó algunos de los temas de los años sesenta: técnicas de muestreo, construcción de cuestionarios, entrevistas, observación participante, investigación-acción, etc. También recuperó tópicos básicos de lógica de la investigación.

Uno de los temas que ha animado la discusión metodológica en América Latina es la diferencia entre los métodos cualitativos y los cuantitativos, que do-

6 Aunque la inestabilidad también afectó a la economía chilena, tal vez este país fue la excepción en la medida en que su economía creció durante estos años. 
minó el escenario de la metodología en las décadas de los ochenta y los noventa, y que aún es pasto de enfrentamientos en algunos países de América Latina. La discusión metodológica sobre este tema, abordada desde la conceptuación piagetiana, hace ver que la incompatibilidad no se puede originar en las características diferenciales del objeto de conocimiento (lo que sería una manera de introducir al empirismo por la ventana), ni en diferencias en los sujetos, aún más, ni siquiera en diferencias esenciales de los conceptos usados por las distintas teorías (Cortés, 2002). Por ello, se ha sostenido que la diferencia es táctica, pero no estratégica (King, Keohane, y Verba, 2000).

En los últimos años hemos asistido a la discusión de conceptos tales como exclusión social, vulnerabilidad, marginación, cohesión social, capital social, integración, equidad y algunos más. En la actualidad, en nuestros países está en el tapete de la controversia la identificación, tamaño y evolución de las clases sociales, el comportamiento de la movilidad social, las tendencias de la desigualdad y de la pobreza, así como el combate al trabajo informal. Estos son los temas que preocupan a una corriente de pensamiento impulsada desde Estados Unidos que trata de avalar la idea de que el modelo económico dominante empieza a rendir frutos debido a que en los países de América Latina la desigualdad en la distribución del ingreso ha empezado a declinar (Lustig, y López, 2010), y la sociedad se ha hecho más fluida y las clases medias han tendido a crecer (Ferreira et al., 2013), lo que significaría que la pobreza habría decaído, pero que es necesario profundizar las reformas económicas para abatir el peso del sector informal que impide el crecimiento de la productividad y, por esa vía, de la economía (McKinsey, 2014).

El estado de la cuestión es una consecuencia lógica de la tendencia a discutir conceptos aislados de su contexto teórico. No fue así en el pasado: por ejemplo, las disputas en torno a la marginalidad que tuvieron lugar a finales de los ańos sesenta y comienzos de los setenta se libraron en el marco de la teoría de la modernización y del marxismo académico, explícitamente vinculados a dos proyectos políticos antagónicos (Cortés, 2000). Debemos tomar conciencia de que detrás de las diversas perspectivas teóricas hay una carga ideológica no explícita en relación con el modelo de desarrollo y la política social (Yocelevzky, 2013).

La metodología de las ciencias enseña que los conceptos pueden ser vagos o precisos, aplicables o inaplicables, iluminadores o no para organizar la experiencia, estériles o fecundos, pero no pueden ser verdaderos o falsos; sólo los enunciados, proposiciones o hipótesis pueden serlo. El sentido de un enunciado teórico no enraíza en su falsación sino está dado por las proposiciones antecedentes y por las que se derivan de él (Bunge, 1999: 83-95), lo que es equivalente a plantear que el sentido de los conceptos y las proposiciones está dado por la teoría en 
que se hallan situados. El significado pleno de un enunciado está dado no sólo por el sentido sino también por su clase de referencia, que equivale a la colección de objetos contenidos en los dominios de los predicados que forman parte de la proposición.

La reducción de los enfrentamientos "teóricos" a conceptos desligados de los cuerpos teóricos que le dan significado, y su limitación al campo de los indicadores, lleva a que los argumentos se envuelvan en un hálito de "objetividad" dentro del cual pareciera que las teorías y las posiciones político-ideológicas no intervinieran, y que el problema central se reduce a discusiones técnicas circunscritas a las características que deben satisfacer los buenos indicadores o índices.

Si se examinan, a modo de ejemplo, los planteamientos sobre las clases medias encontramos que, desde la economía, se plantea que la estimación del tamaño de la clase media se puede abordar por dos caminos: i) clasificando como clase media a los hogares cuyo ingreso (absoluto) cae dentro de un intervalo previamente definido, ii) o bien, determinando que dicha clase se conforma por los hogares localizados en percentiles preestablecidos de la distribución del ingreso (relativo).

Desde la primera de estas perspectivas basta con identificar los valores de ingreso mínimo (que debe ser superior al de pobreza) y máximo (elevado pero inferior al de riqueza), para determinar si un hogar o una persona pertenece o no a la clase media. Para Banerjee, y Dufflo (2007), ésta se encontraría formada por los miembros de los hogares que tienen un ingreso per cápita diario de entre dos y diez dólares, mientras que para Ravallion (2009) el rango estaría entre los dos y los trece dólares diarios. ${ }^{7}$

Con la intención de disminuir la arbitrariedad en la determinación del valor de los umbrales mínimo y máximo que limitan a las clases medias, López, y Ortiz (2011) han propuesto emplear el concepto de vulnerabilidad, en tanto que Cruces, López y Battistón (2011) el de polarización que, en combinación con criterios estadísticos, permiten identificar los puntos de corte de la distribución. También se ha empleado como cota inferior la vulnerabilidad a la pobreza (Ferreira et al., 2013: 2).

Quienes han seguido el segundo camino suelen definir como cota inferior de clase media el segundo o tercer deciles, y como cota superior el quintil o decil más alto de la distribución del ingreso. La idea es que aquellos hogares cuyos

\footnotetext{
Los autores usan dólares expresados en la misma capacidad adquisitiva, lo que permite las comparaciones internacionales (para uniformar los valores expresados en las monedas nacionales, se usa el tipo de cambio de Paridad del Poder Adquisitivo o en inglés Purchasing Power Parity). Ambos procedimientos de estimación coinciden en la cota mínima de US $\$ 2$, que a su vez coincide con el valor de la línea de pobreza que usa el Banco Mundial para América Latina.
} 
ingresos están por debajo del umbral mínimo no son de la clase media por ser pobres y los que están por encima del umbral superior, tampoco, por tener un ingreso muy elevado. ${ }^{8}$ Ahora bien, los umbrales suelen ser distintos para diferentes autores; así, por ejemplo, para Barro (1999) y para Easterly (2001) la clase media estaría formada por los hogares del cuarto al sexto deciles, mientras que para Solimano (2008), por los del tercero al noveno deciles de hogares, ordenados según el ingreso per cápita. Nótese que si estos diversos criterios se aplicaran a una misma distribución de ingresos se obtendrían tantas clases medias como definiciones. Birdsall (2010) emplea un método híbrido: el umbral inferior es de diez dólares por día y el superior, el percentil de ingreso 95 .

Detrás de esta forma de cuantificar el tamaño de las clases medias y su evolución, subyace la teoría del bienestar. La versión más estrecha de esta teoría plantea que el bienestar de las personas depende de su consumo de bienes y servicios, el cual estaría determinado por el ingreso y los precios de mercado. Según esta teoría, habría un nivel de bienestar tal que cualquier persona que no lo alcanzara sería pobre, las personas de clase media estarían por encima de ese nivel, y en la distribución del bienestar habría un punto superior a partir del cual finaliza la clase media e inicia la clase alta. La discusión sobre los indicadores hace borrosa la teoría subyacente, su forma de concebir lo real y su movimiento.

Siguiendo con el tema, De la Calle y Rubio, en una publicación que ha tenido amplias reverberaciones en diversos círculos de México, consideran que los sectores medios estarían conformados por personas que viven en hogares con un nivel de ingreso medio, cuyo jefe tiene escolaridad de preparatoria, un automóvil y posibilidad de realizar un viaje al año; y por personas con ingreso un poco por debajo del ingreso medio, con educación secundaria y sin automóvil. ${ }^{9}$ Estos mismos autores sostienen que: "En suma, la clase media se puede concebir como un conjunto de estratos diferenciados cuyas características comunes son esencialmente culturales, de actitud y de patrones de consumo" (De la Calle, y Rubio, 2010: 18). Ahora bien, en este caso al bienestar económico (medido por gasto) se adicionan los valores y las actitudes. Y agregan que "para académicos muy formales, esta caracterización es sin duda imprecisa, pero para analistas de fenómenos políticos y estrategas electorales - $\mathrm{y}$ para no pocos expertos en mercadotecnia-, estos elementos pueden hacer la diferencia entre ganar y perder una elección, o entre un negocio viable y uno

8 Por construcción, este camino para definir la clase media no permite registrar su evolución a lo largo del tiempo, porque se define a partir de una proporción que no se modifica.

9 Sus estimaciones se basan en los estratos sociales construidos por la Asociación Mexicana de Agencias de Investigación de Mercados y de Opinión Pública (AMAI). 
que no lo es" (De la Calle, y Rubio, 2010: 17-18). Nuevamente, lo central son los indicadores aunque también esta propuesta descansa en la teoría económica del bienestar y en el agregado de los valores.

El Instituto Nacional de Estadística y Geografía (INEGI) también ha terciado en la discusión y ha calculado que la clase media agrupaba al $39.1 \%$ de las personas en el año 2010 y alcanzaba al 35.2\% de la población en el 2000.

El cálculo se realizó empleando técnicas de aglomeración multidimensional, utilizando 17 variables de las correspondientes Encuestas Nacional de Ingresos y Gastos. Cabe resaltar que en este estudio nunca se tiene que prejuzgar cuáles son los umbrales de nivel de vida (sean en términos de gasto o de ingresos) que separan a las clases sociales ni tampoco los porcentajes que deben haber en ellas de personas con escolaridad o en la jerarquía ocupacional del mercado laboral. Simplemente los resultados de la estratificación a los que se llega sugieren cuántos y cuáles son los niveles a tomar en cuenta y el análisis ulterior verifica en dónde se detectan diferencias cualitativas (instrucción, posición e inserción laboral y acceso a la propiedad), tras las diferencias meramente cuantitativas (gastos o ingresos). En esto la presente investigación es radicalmente diferente — desde el punto de vista metodológico- a cualquier otra que se ha emprendido con respecto a la información referida a México ya que no requiere de acotamientos de gasto o de ingreso fijados de antemano o establecidos como una precondición antes de clasificar los hogares. Dicho de otra manera, las fronteras que se identifican entre los estratos para determinar en cuál de ellos comienza y en cuál otro termina la clase media son un resultado al que se llega no una premisa de la que se depende en este estudio (INEGI, 2013).

Como se aprecia en esta cita, de nuevo el énfasis está puesto en los indicadores y en la técnica utilizada para "hacer hablar a los datos" (Yaschine, 2014), manteniendo la apariencia de neutralidad. Hemos vuelto a la noción que creíamos superada de que el dato está dado.

Ahora bien, en contraposición con estas aproximaciones modernas a las clases sociales, hay que recordar que la división de la sociedad en clases ha sido una de las preocupaciones centrales de la sociología. En esta disciplina se observa con claridad que el concepto adquiere sentidos distintos en teorías diferentes. Según Marx, en el modo de producción capitalista, la propiedad de los medios de producción divide a la población activa en propietarios (burgueses o capitalistas) y no propietarios (trabajadores o proletarios) que sólo disponen de su fuerza de trabajo. Sin embargo, el mismo Marx reconoce (en su tratamiento de la plusvalía) que el propio desarrollo del capitalismo generaría la necesidad de actividades de control y, por lo tanto, de puestos de gerentes y supervisores, 
y para asegurar la circulación del capital, de trabajadores "improductivos" como servidores públicos, trabajadores de las finanzas y la banca. Esta parte de la teoría marxista ha quedado sepultada por la visión de la sociedad organizada en torno a las dos clases fundamentales (Benza, 2012: 21).

Por otra parte, para comprender cabalmente la noción de clase expuesta por Weber, es necesario precisar algunos conceptos. La situación de clase es una forma de división social que refleja las relaciones de poder en la esfera mercantil, y agrupa a los individuos con una situación de mercado común en función de sus bienes y sus capacidades. El volumen, tasas de usos y precios de mercado de los activos físicos, financieros y de sus capacidades laborales valorizables en el mercado, determinan sus oportunidades de vida, entendidas como el conjunto de probabilidades típicas de provisión de bienes, de posición externa a sus fuentes de ingreso y de destino personal. En síntesis, la situación de clase va más allá de los individuos y está conformada por las relaciones de producción y de adquisición en el mercado. La clase social corresponde a un conjunto de situaciones de clase, pero por su movilidad social inter e intrageneracional se acercan a un estamento.

El funcionalismo estructural emplea la noción de estrato en lugar del concepto de clase social, que al formar parte de una pirámide social da la idea de un continuo que atraviesa desde la base hasta la cúspide. De acuerdo con esta concepción, los roles ocupacionales que gozarían de mayor estimación social serían los que mayor importancia tendrían para la reproducción del sistema social. Como consecuencia, la jerarquía de reconocimientos y retribuciones serían poderosos alicientes para que los individuos más talentosos ocupen las posiciones más importantes garantizando la eficiencia en el funcionamiento del sistema.

Esta concepción suponía que la evolución de las sociedades industriales aumentaría constantemente los empleos que requieren de personal técnico y profesional altamente calificado, y que este proceso estaría acompañado por una alta movilidad social. Como consecuencia de ambos procesos, tendían a eliminarse las diferencias de clase limando así una de las causas de la conflictividad.

\section{Conclusión}

El recuento que se ha presentado sobre la metodología de las ciencias sociales vis à vis las diversas posiciones teóricas y los proyectos de sociedad en los últimos cincuenta años, permite ver que su contenido actual recoge las capas que se fueron agregando a lo largo del tiempo. A través de las denominadas técnicas de encuesta (survey) predominantes en los inicios, la metodología se 
vinculaba estrechamente con la estadística al punto que los libros de texto más utilizados solían incluir algunos temas estadísticos. El enfrentamiento paradigmático de los años sesenta y parte de los setenta trajo el predominio de las preocupaciones epistemológicas y los intentos de establecer los vínculos con la investigación empírica. En los tiempos que transcurren, los problemas metodológicos se vuelven a relacionar estrechamente con los desarrollos de la estadística, disciplina que, junto a los avances de la computación y la disponibilidad de poderosos paquetes de programas de cómputo, proporciona los instrumentos para trabajar grandes bases de datos, que contienen muchos casos y un gran número de variables.

El campo de la metodología —entendida como el conjunto de operaciones que permiten ligar las teorías con la evidencia (observación, experimentación, medición, diseños de investigación) — parece perder terreno en la medida en que se impone la idea de construir teorías a partir de los indicadores y de los datos. La estrategia de investigar "pescando" regularidades en los datos, hoy se ve facilitada por la creciente disponibilidad de información y las facilidades de procesamiento. No es novedosa en absoluto, fue el punto de partida del empirismo lógico, pero también su final, pues nunca se pudo generar evidencia para sostener empíricamente que la realidad es directamente observable (Ayer, 1965: 19). El ejemplo de las clases sociales que presentamos en la cuarta sección es útil también para ilustrar que la elección de las variables, así como el tratamiento que se da a los datos, están influidos por decisiones que toma el investigador. Aunque profese y aplique criterios de "objetividad", sus resultados variarán de acuerdo con las opciones que seleccionó. ¿Por qué elegir la variable ingreso para hacer observable el concepto clase social? ¿Por qué no el gasto? ¿La posesión de automóvil? ¿La posición en la ocupación? ¿El nivel educativo? Una vez que el investigador ha seleccionado una o más variables, ¿qué tratamiento les dará? Ya vimos que en el caso más simple de elegir un sólo indicador empírico (el ingreso) se pueden generar varias soluciones de acuerdo a los criterios que se decide emplear para establecer la relación entre los valores de las variables y las clases sociales.

Por último, el recuento que se ha presentado a lo largo de este texto muestra que la aplicación adecuada de conceptos básicos de la metodología permite trazar el derrotero que debe seguirse para, a partir de las variables empleadas, develar las teorías implícitas, además de intervenir en las discusiones técnicas sobre indicadores y su tratamiento estadístico. Una vez que se identificó el cuerpo teórico que orientó la investigación, es posible inferir su correspondiente paquete de valores, lo que permite contrastar esos resultados con los que presentan otras investigaciones y, de ser el caso, proceder a la discusión crítica con fundamento. 


\section{Referencias}

Aibar, Julio, Fernando Cortés, Liliana Martínez, y Gisela Zaremberg (coords.), 2013, El helicoide de la investigación. Metodología en tesis de ciencias sociales, México, Flacso México.

Ayer, Alfred Jules, 1965, El positivismo lógico, México, Fondo de Cultura Económica.

Alesina, Alberto, y Roberto Perotti, 1996, "Income Distribution, Political Instability, and Investment”, European Economic Review, núm. 40.

Banerjee, Abhijit, y Esther Duflo, 2008, "What is Middle Class about the Middle Classes around the World?", Massachusetts Institute of Technology, Department of Economics, Working Paper núm. 07-29.

Barro, Robert, 1999, "Determinants of democracy", Journal of Political Economy, 107(S6), pp. 158-183.

Benza, Gabriela, 2012, Estructura de clases y movilidad intergeneracional en Buenos Aires: ¿el fin de una sociedad de "amplias clases medias"?, tesis de doctorado, México, El Colegio de México, Centro de Estudios Sociológicos.

Birdsall, Nancy, 2010, The (Indispensable) Middle Class in Developing Countries; or, the Rich and the Rest, Not the Poor and the Rest, Working Paper 207, Center for Global Development.

Blalock, Hubert, 1961, Causal Inference in Nonexperimental Research, Chapel Hill, The University of North Carolina Press.

Boudon, Raymond, 1970, L’analyse mathématique des faits sociaux, París, Plon.

Bunge, Mario, 1999, Buscar la filosofía en las ciencias sociales, México, Siglo XXI.

Cortés, Fernando, 2002, "Marginalidad, marginación, pobreza y desigualdad en la distribución del ingreso", Papeles de Población, año 8, núm. 31, enero-marzo.

Cortés, Fernando, 2000, "Algunos aspectos de la controversia entre investigación cualitativa e investigación cuantitativa”, Argumentos: Estudios críticos de la sociedad, núm. 36, División de Ciencias Sociales y Humanidades, UAM-Xochimilco.

Cortés, Fernando, y Rosa María Rubalcava, 1987, Métodos estadísticos aplicados a la investigación social en ciencias sociales: análisis de asociación, México, El Colegio de México.

Cortés, Fernando, Adam Przeworski, y John Sprague, 1974, System Analysis for Social Scientists, New York, John Wiley. 
Cortés, Fernando, y Adam Przeworski, 1971, "Sistemas partidistas, movilización electoral y la estabilidad en sociedades capitalistas", Revista Latinoamericana de Ciencias Politicas y Administrativas, vol. II, Flacso.

Cortés, Fernando, y Manuel Castells, 1968, "Análisis causal y técnicas estadísticas en la investigación sociológica”, documento interno de la Flacso Chile.

Cruces, Guillermo, Luis Felipe López Calva, y Diego Battistón, 2011, Down and Out or Up and In? Polarization-Based Measures of the Middle Class for Latin America, Documento de Trabajo núm. 113, Centro de Estudios Distributivos, Laborales y Sociales (CEDLAs), Universidad Nacional de La Plata.

De la Calle, Luis, y Luis Rubio, 2010, Clasemediero: Pobre no más, desarrollado aún no, México, Centro de Investigación para el Desarrollo.

Easterly, William, 2001, “The middle class consensus and economic development”, Journal of Economic Growth, vol. 6, núm. 4, pp. 317-335.

Ferreira, Francisco, Julián Messina, Jamele Rigolini, Luis Felipe López Calva, María Ana Lugo, y Renos Vakis, 2013, La movilidad económica y el crecimiento de la clase media en América Latina, Washington, DC, Banco Mundial.

Galtung, Johan, 1966, Teoría y métodos de la investigación social, Buenos Aires, Editorial Universitaria de Buenos Aires.

García, Rolando, 2000, El conocimiento en construcción. De las formulaciones de Jean Piaget a la teoría de los sistemas complejos, Barcelona, Gedisa.

INEGI, 2013, Cuantificando la clase media en México: un ejercicio exploratorio, disponible en <http://goo.gl/P8LMm8>, consultado el 22 de junio de 2014.

King, Gary, Robert Keohane, y Sidney Verba, 2000, El diseño de la investigación social: la inferencia cientifica en los estudios cualitativos, Madrid, Alianza Editorial.

Lazarsfeld, Paul, 1974, "La interpretación de las relaciones estadísticas como propiedad de investigación”, en Boudon Raymond, Metodología de las ciencias sociales II. Análisis empirico de la causalidad, Barcelona, Laia.

López Calva, Luis Felipe, y Nora Lustig, 2010, "Explaining the Decline in Inequality in Latin America: Technological Change, Educational Upgrading, and Democracy”, en N. Lustig, y L. F. López Calva (eds.), Declining Inequality in Latin America: A decade of Progress?, Undp/ Brookings Institution Press. 
López Calva, Luis Felipe, y Eduardo Ortiz Juárez, 2011, A Vulnerability Approach to the Definition of Middle Class, Policy Research Working Paper Series 5902, The World Bank.

McKinsey Global Institute, 2014, A Tale of Two Mexicos: Growth and prosperity in a two-speed economy, Londres, McKinsey Co.

Piaget, Jean, 1978, La equilibración de las estructuras cognitivas. Problema central del desarrollo, Madrid, Siglo XXI.

Piaget, Jean, y Rolando García, 1982, Psicogénesis e historia de la ciencia, México, Siglo XXI.

Popper, Karl, 1962, La lógica de la investigación científica, Tecnos, Madrid.

Prigogine, Ilya, e Isabelle Stengers, 1979, La nueva alianza: metamorfosis de la ciencia, Madrid, Alianza Editorial.

Przeworski, Adam, y Henry Teune, 1972, The Logic of Comparative Social Inquiry, Nueva York, John Wiley.

Ravallion, Martin, 2009, The Developing World's Bulging (but Vulnerable) "Middle Class", Policy Research Working Paper 4816, World Bank.

Simon, Herbert, 1957, Models of Man, Nueva York, John Wiley.

Solimano, Andrés, 2008, "The Middle Class and the Development Process: International Evidence”, Macroeconomía del desarrollo, núm. 11, Economic Commission for Latin America and the Caribbean (ECLAC).

Williamson, John, 1990, "What Washington Means by Policy Reform”, en John Williamson (ed.), Latin American Adjustment. How Much Has Happened?, Washington, DC, Institute for International Economics.

Williamson, John, 2003, "An Agenda for Restarting Growth and Reform”, en Pedro Pablo Kuczynski, y John Williamson (eds.), After the Washington Consensus: Restarting Growth and Reform in Latina America, Washington, DC, Institute for International Economics.

Wright, Sewall, 1934, “The Methods of Path Coefficients", The Annals of Mathematical Statistics, vol. 5 , núm. 3 .

Yaschine, Iliana, 2013, "Comentarios al informe del INEgr sobre clases medias", México Social, año 2 , núm. 36, julio, pp. 6-7. 
Yocelevzky, Ricardo, 2013, "Notas preliminares para una discusión acerca de las clases medias", Les Cahiers de Psychologie Politique (en línea), núm. 23, disponible en <http://goo.gl/ yyHWaM>.

Recibido el 1 de septiembre de 2014. Aceptado el 25 de septiembre de 2014. 